\title{
Time-dependent reversal of significant intrapul- monary shunt after liver transplantation
}

Xin Jin ${ }^{1}$, Byung Joo Sun ${ }^{1}$, Jae-Kwan Song ${ }^{1}$, Jae-Hyung Roh ${ }^{1}$, Jeong Yoon Jangㄹ, Dae-Hee Kim, Young-Suk Lim ${ }^{2}$, Jong-Min Song ${ }^{1}$, Duk-Hyun Kang ${ }^{1}$, and Sung Gyu Lee ${ }^{3}$

${ }^{1}$ Cardiac Imaging Center, Departments of ${ }^{2}$ Gastroenterology and ${ }^{3}$ Surgery, Asan Medical Center, University of Ulsan College of Medicine, Seoul, Korea
Received: March 28, 2017

Revised : August 17, 2017

Accepted: September 18, 2017

\section{Correspondence to}

Jae-Kwan Song, M.D.

Cardiac Imaging Center, Asan Medical Center, University of Ulsan College of Medicine, 88 Olympic-ro 43-gil, Songpa-gu, Seoul 05505, Korea Tel: +82-23010-3155

Fax: +82-2-486-5918

E-mail:jksong@amc.seoul.kr
Background/Aims: Although the association between intrapulmonary shunt (IPS) and liver cirrhosis is clear, data of repeated contrast echocardiography (CE) before and after liver transplantation (LT) to evaluate factors associated with IPS are limited.

Methods: Hand-agitated saline was used for CE and, by assessing left-chamber opacification, IPS was classified as grade o to 4 . Grade 3/4 constituted significant IPS and hepatopulmonary syndrome (HPS) was defined as significant IPS with the arterial partial pressure of oxygen $<70 \mathrm{mmHg}$.

Results: Before LT, 253 patients underwent CE and the frequency of significant IPS and HPS were $44 \%(\mathrm{n}=112)$ and $7 \%(\mathrm{n}=17)$, respectively. Child-Pugh score (odds ratio [OR], 1.345; 95\% confidence interval [CI], 1.090 to 1.66o; $p=0.006$ ) and arterial oxygen content (OR, $0.838 ; 95 \% \mathrm{CI}, 0.708$ to $0.991 ; p=0.039)$ were independent determinants of significant IPS, whereas direct bilirubin (OR, 1.076; 95\% CI, 1.012 to $1.144 ; p=0.019$ ) was the only variable associated with HPS. Among 153 patients who underwent successful LT, repeated CE was performed in 97 (63\%), which showed significant reductions in IPS grade (from $2.6 \pm 1.0$ to $1.2 \pm 1.3, p<$ 0.001 ) and the prevalence of significant IPS (from $56 \%$ to $20 \%, p=0.038$ ). After adjustment for pre-LT IPS grade, time from LT to repeated CE presented negative linear relationship with post-LT IPS grade $\left(r^{2}=0.366, p<0.001\right)$ and was the only determinant of post-LT IPS grade (OR, 1.009; 95\% CI, 1.003 to $1.014 ; p=0.004$ ).

Conclusions: Repeated CE is useful to evaluate intrapulmonary vascular change before and after LT. Reversal of IPS after successful LT is time-dependent and follow-up duration should be considered for accurate assessment of IPS after LT.

Keywords: Liver cirrhosis; Liver transplantation; Intrapulmonary shunt; Echocardiography

\section{INTRODUCTION}

Hepatopulmonary syndrome (HPS) represents pathological pulmonary vascular alterations in patients with end-stage liver cirrhosis (LC) and significant intrapulmonary shunt (IPS) is believed to be a cause of this potentially fatal complication [1-3]. Contrast-enhanced transthoracic echocardiography using hand-agitated saline (cTTE) is a well-established diagnostic method for detection of IPS [4-6]. The cTTE has been used for clinical investigation to evaluate the incidence and risk factors associated with development of significant IPS [7-10]. It has been reported that successful liver transplantation (LT) can result in complete reversal of sig- 
nificant IPS or HPS [11-13]. However, these observations are based on a few clinical studies with relatively small numbers of patients or case reports. Systematic clinical investigations using follow-up cT'TE after successful LT for a relatively large numbers of patients are limited. We investigated the outcome of significant IPS after LT and evaluated the factors determining significant IPS before and after successful LT.

\section{METHODS}

\section{Patients}

From May 2012 to April 2015, all eligible LT candidates were prospectively screened at Asan Medical Center. According to the protocol approved by our Institutional Review Board (2018-0100), patient eligibility was assessed and written informed consent was obtained from all patients. We included all patients with LC and adequate echocardiographic quality. Patients with intracardiac shunt, symptomatic heart failure, active pneumonia, or significant lung disease were excluded. A total of 253 patients including 123 patients with hepatocellular carcinoma were included in this analysis.

\section{Contrast echocardiography}

All patients underwent cT'TE using Philips IE 33 system (Philips Medical Systems, Andover, MA, USA) equipped with a $2.5-\mathrm{mHz}$ transducer. A baseline two-dimensional apical four-chamber view was acquired and hand-agitated saline was used for cTTE. Intravenous access with a three-way stopcock was made on the antecubital vein before examination. To generate micro-bubbles, two sterile syringes filled with normal saline and air (8 and 2 $\mathrm{mL}$, respectively) were connected through the three-way stopcock and pushed alternatively for 10 to 15 seconds. A transducer was placed in a stable position for $>15$ seconds after injection of hand-agitated saline and repeated injections of hand-agitated saline were performed to acquire adequate image contrast. The presence of IPS was defined as the appearance of a microbubble in the left atrium (LA) during three to six cardiac cycles after opacification of the right atrium.

Depending on the degree of left heart chamber opacification, IPS was classified as grade o (none), grade 1 (small amount of bubbles within LA), grade 2 (bubbles occupying half of the LA), grade 3 (bubbles occupying more than half of the LA), and grade 4 (bubbles occupying both LA and left ventricle) (Fig. 1). Patients with grade 3 or 4 IPS were defined to have significant IPS.

\section{Grade 0} Grade 1

Grade 2

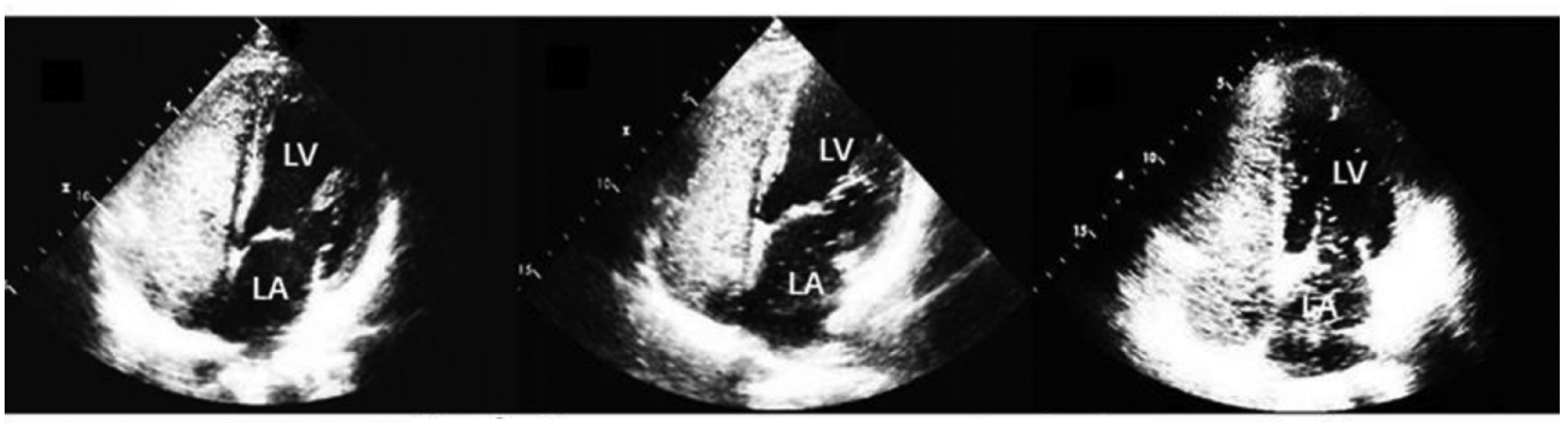

Grade 3

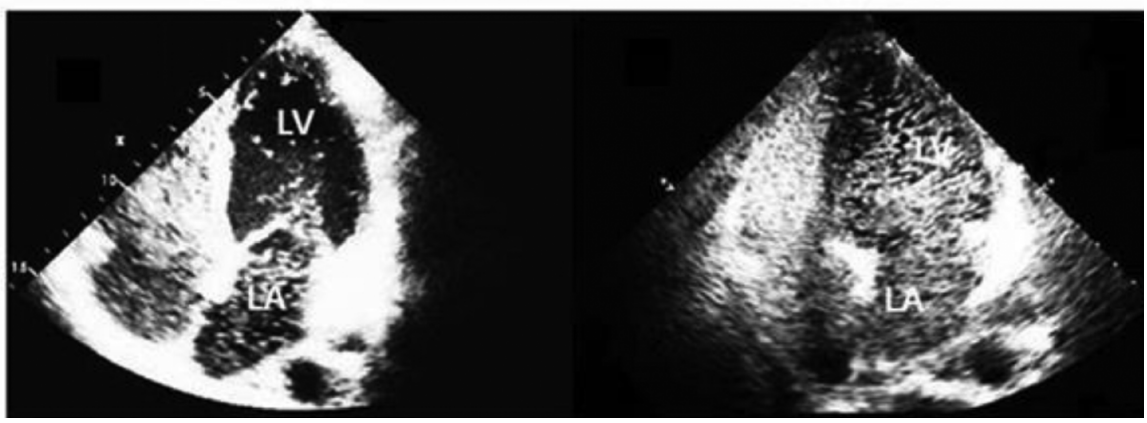

Figure 1. Representative echocardiographic images showing different intrapulmonary shunt severity on contrast-enhanced transthoracic echocardiography. LV, left ventricle; LA, left atrium. 
Table 1. Comparison of clinical features between patients with and without significant IPS

\begin{tabular}{|c|c|c|c|}
\hline Variable & $\operatorname{IPS}(+)(n=112)$ & $\operatorname{IPS}(-)(n=141)$ & $p$ value \\
\hline Age, yr & $53 \pm 10$ & $56 \pm 9$ & 0.013 \\
\hline Etiology of liver cirrhosis, & & & 0.694 \\
\hline $\mathrm{HBV}$ & $73(65)$ & $89(63)$ & \\
\hline $\mathrm{HCV}$ & $11(10)$ & $18(13)$ & \\
\hline Alcoholic & $19(17)$ & $19(13)$ & \\
\hline Non-viral/Non-alcoholic & $9(8)$ & $15(11)$ & \\
\hline Hepatocelluar carcinoma & $53(47 \cdot 3)$ & $70(49.6)$ & 0.800 \\
\hline Hemoglobin, g/dL & $10.9 \pm 2.1$ & $11.9 \pm 2.2$ & $<0.001$ \\
\hline Platelet, $10^{3} / \mathrm{mm}^{3}$ & $74 \pm 42$ & $87 \pm 50$ & 0.020 \\
\hline Child-Pugh score & $9.1 \pm 2.2$ & $7 \cdot 5 \pm 2.2$ & $<0.001$ \\
\hline Prothrombin time, INR & $1.6 \pm 0.5$ & $1.4 \pm 0.4$ & $<0.001$ \\
\hline Albumin, g/dL & $2.8 \pm 0.6$ & $3.1 \pm 0.7$ & $<0.001$ \\
\hline Total bilirubin, mg/dL & $6.2 \pm 8.8$ & $3.6 \pm 6.5$ & 0.010 \\
\hline Direct bilirubin, mg/dL & $4.2 \pm 7.1$ & $2.2 \pm 5.2$ & 0.017 \\
\hline $\mathrm{ALT}, \mathrm{U} / \mathrm{L}$ & $40.7 \pm 48.4$ & $41.0 \pm 61.9$ & 0.963 \\
\hline LV ejection fraction, \% & $65 \pm 4$ & $64 \pm 4$ & 0.065 \\
\hline Peak TR velocity, m/sec & $2.3 \pm 0.3$ & $2.3 \pm 0.3$ & 0.924 \\
\hline $\mathrm{PaO}_{2}, \mathrm{mmHg}$ & $76.4 \pm 13.2$ & $83.6 \pm 11.2$ & 0.002 \\
\hline Arterial $\mathrm{O}_{2}$ saturation, $\%$ & $97 \cdot 4 \pm 2.3$ & $98.1 \pm 1.7$ & 0.015 \\
\hline $\mathrm{CaO}_{2}, \mathrm{~mL} / \mathrm{dL}$ & $13.9 \pm 2.4$ & $15.9 \pm 2.9$ & $<0.001$ \\
\hline
\end{tabular}

Values are presented as mean \pm SD or number (\%).

IPS, intrapulmonary shunt; HBV, hepatitis B virus; HCV, hepatitis C virus; INR, international normalized ratio; ALT, alanine aminotransferase; LV, left ventricle; TR, tricuspid regurgitation; $\mathrm{PaO}_{2}$, arterial partial pressure of oxygen; $\mathrm{CaO}_{2}$, arterial oxygen content.

All the echocardiographic images were reviewed by two independent experienced cardiologists (X.J. and J.K.S.). IPS severity was determined on the basis of the consensus of these two reviewers. In addition, HPS was defined as significant IPS with the arterial partial pressure of oxygen $<70 \mathrm{mmHg}$.

\section{Statistical analysis}

Categorical variables were presented as numbers and percentages and continuous variables were expressed as mean \pm standard deviation (SD). Variables were compared using the Fisher exact test or Student $t$ test, as appropriate. Binary logistic regression models were used to assess the independent factors associated with the presence of significant IPS and HPS before and after LT. Spearman's rank correlation coefficient was used to identify and test the strength of the relationship between Child-Pugh score and IPS severity before LT.
Linear regression was used to study the relationship between post-LT IPS severity and follow-up duration, which was adjusted for pre-LT IPS grade. Kaplan-Meier analysis with log rank tests were used for the comparison of survival curves. Cox regression analysis was used to find factors associated with survival in patients who did not undergo LT. A $p$ value of $<0.05$ was considered statistically significant. SPSS version 20 (IBM Co., Armonk, NY, USA) was used for statistical analysis.

\section{RESULTS}

\section{Contrast echocardiography before LT}

A total of 253 patients underwent baseline cTTE. IPS of any grade was present in 212 patients (84\%), and 112 (44\%) had significant IPS (grade 3/4 = 66/46). Patients with significant IPS were characterized by a higher Child-Pugh 
score (9.1 \pm 2.2 vs. $7.5 \pm 2.2, p<0.001)$, lower age $(53 \pm 10$ years vs. $56 \pm 9$ years, $p=0.013$ ), longer prothrombin time (1.6 \pm 0.5 vs. $1.4 \pm 0.4, p<0.001)$, lower hemoglobin levels $(10.9 \pm 2.1 \mathrm{~g} / \mathrm{dL}$ vs. $11.9 \pm 2.2 \mathrm{~g} / \mathrm{dL}, p<0.001)$, lower platelet levels (74 $\pm 4210^{3} / \mathrm{mm}^{3}$ vs. $\left.87 \pm 5010^{3} / \mathrm{mm}^{3}, p=0.020\right)$, lower albumin levels $(2.8 \pm 0.6 \mathrm{~g} / \mathrm{dL}$ vs. $3.1 \pm 0.7 \mathrm{~g} / \mathrm{dL}, p<$ $0.001)$, higher total bilirubin levels $(6.2 \pm 8.8 \mathrm{mg} / \mathrm{dL}$ vs. 3.6 $\pm 6.5 \mathrm{mg} / \mathrm{dL}, p=0.010)$, and lower arterial oxygen content $\left(\mathrm{CaO}_{2}\right)(13.9 \pm 2.4 \mathrm{~mL} / \mathrm{dL}$ vs. $15.9 \pm 2.9 \mathrm{~mL} / \mathrm{dL}, p<0.001)$ than those without significant IPS (Table 1). A significant positive relationship was found between Child-Pugh score and IPS severity $(p<0.001)$ (Fig. 2$)$. In the univariate analysis, age, Child-Pugh score, albumin, total and direct bilirubin, and $\mathrm{CaO}_{2}$ were associated with the presence of significant IPS. Among them, Child-Pugh score (odds ratio [OR], 1.345; 95\% confidence interval [CI], 1.090 to $1.660 ; p=0.006)$ and $\mathrm{CaO}_{2}(\mathrm{OR}, 0.838 ; 95 \% \mathrm{CI}, 0.708$ to $0.991 ; p=0.039)$ were confirmed to be independent variable associated with significant IPS (Table 2).

Before LT, arterial blood gas analysis was done in 115 patients and HPS was present in 17 patients (14.8\%). Patients with HPS were characterized by prolonged prothrombin time $(1.8 \pm 0.7$ vs. $1.5 \pm 0.5, p=0.048)$, lower albumin level $(2.7 \pm 0.3 \mathrm{~g} / \mathrm{dL}$ vs. $2.9 \pm 0.6 \mathrm{~g} / \mathrm{dL}, p=0.022)$, higher Child-Pugh score ( $9.8 \pm 2.0$ vs. $8.7 \pm 2.3, p=0.053)$, and higher direct bilirubin $(8.2 \pm 11.4 \mathrm{mg} / \mathrm{dL}$ vs. $3.2 \pm 5.7$ $\mathrm{mg} / \mathrm{dL}, p=0.099)$. Binary logistic regression analysis showed that direct bilirubin level was the only independent factor associated with HPS (OR, 1.076; 95\% CI, 1.012 to $1.144 ; p=0.019$ ) (Table 3 ).

Table 2. Clinical variables associated with the presence of significant intrapulmonary shunt

\begin{tabular}{|c|c|c|c|c|c|}
\hline \multirow{2}{*}{ Variable } & \multicolumn{2}{|c|}{ Unadjusted } & \multicolumn{3}{|c|}{ Adjusted } \\
\hline & OR & $p$ value & OR & $95 \% \mathrm{CI}$ & $p$ value \\
\hline Age & 0.966 & 0.015 & 0.993 & $0.949-1.039$ & 0.752 \\
\hline Child-Pugh score & 1.353 & $<0.001$ & 1.345 & $1.090-1.660$ & 0.006 \\
\hline Platelets & 0.993 & 0.025 & 0.995 & $0.985-1.005$ & 0.344 \\
\hline Albumin & 0.403 & $<0.001$ & 0.925 & $0.330-2.597$ & 0.883 \\
\hline Total bilirubin & 1.047 & 0.011 & 0.984 & $0.931-1.040$ & 0.568 \\
\hline Direct bilirubin & 1.055 & 0.019 & 1.129 & $0.837-1.522$ & 0.427 \\
\hline $\mathrm{CaO}_{2}$ & 0.760 & $<0.001$ & 0.838 & $0.708-0.991$ & 0.039 \\
\hline
\end{tabular}

OR, odds ratio; $\mathrm{CI}$, confidence interval; $\mathrm{CaO}_{2}$, arterial oxygen content.

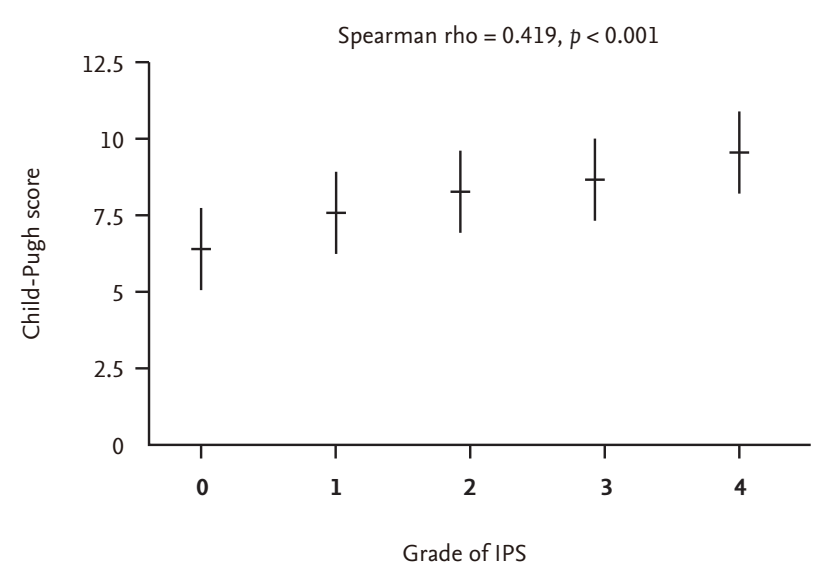

Figure 2. Relationship between Child-Pugh score and intrapulmonary shunt (IPS) severity before liver transplantation.

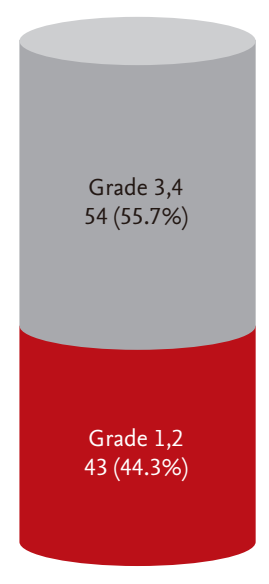

Pre-LT

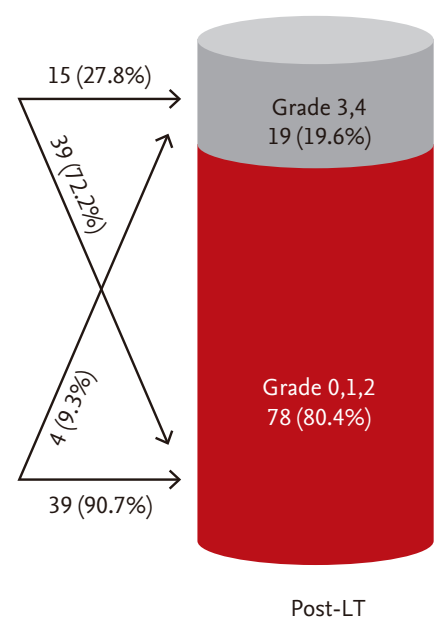

Figure 3. Changes in intrapulmonary shunt grade before and after liver transplantation (LT). 


\section{IPS after LT}

During the study period, a total of 153 patients $(60 \%)$ underwent successful LT, and among them, follow-up cT'TE was performed in 97 (63\%) between 6 and 952 days after LT (median, 255 days). Both IPS grade (from $2.6 \pm$ 1.0 to $1.2 \pm 1.3, p<0.001)$ and the prevalence of significant IPS (from $56 \%$ to $20 \%, p=0.038$ ) decreased significantly after LT (Fig. 3). After adjustment for the pre-LT IPS grade, linear regression revealed a negative relationship between the post-LT IPS grade and follow-up duration $\left(r^{2}=0.366, p<0.001\right)$ (Fig. 4). Among 54 patients with significant IPS before LT, follow-up cTTE showed disappearance of significant IPS in 39 (72.2\%). These patients were characterized by longer follow-up duration (319 \pm 258 days vs. $88 \pm 111$ days, $p<0.001$ ) than those who showed persistent significant IPS. Among patients who did not undergo LT, the survival rate was lower in those with significant IPS, whereas significant IPS did not affect survival in those who underwent LT (Fig. 5). Cox regression analysis confirmed that significant IPS (hazard ratio [HR], 3.432; 95\% CI, 1.096 to $10.748 ; p=0.034$ ) and direct bilirubin (HR, 1.074; 95\% CI, 1.003 to 1.150; $p$ $=0.041$ ) were independently associated with mortality in patients who did not undergo LT (Table 4).

\section{DISCUSSION}

In this study using the data collected in a tertiary referral hospital with a very active LT program, we found that lower $\mathrm{CaO}_{2}$ and higher Child-Pugh score were independent risk factors associated with development of significant IPS before LT. Significant IPS was observed

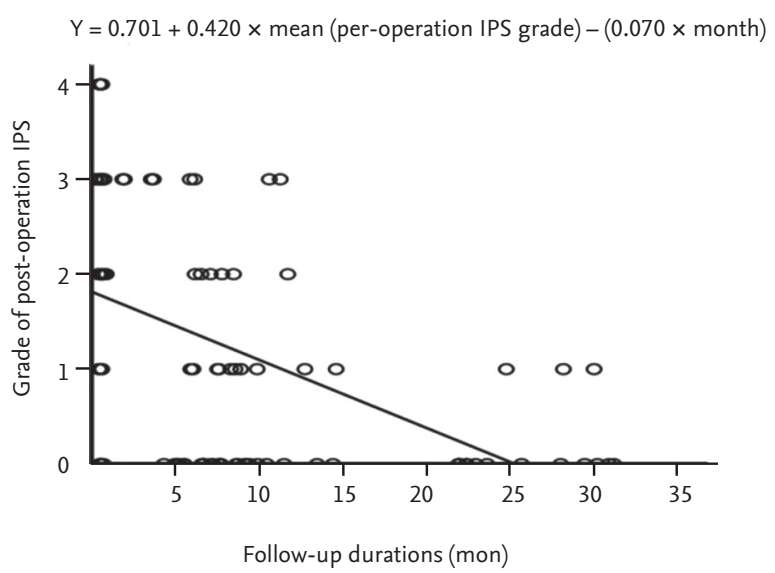

Figure 4. Linear relationship between post-liver transplantation (LT) intrapulmonary shunt (IPS) grade and follow-up duration adjusted by the pre-LT IPS grade.

Table 3. Clinical variables associated with the presence of hepatopulmonary syndrome

\begin{tabular}{|c|c|c|c|c|c|}
\hline \multirow{2}{*}{ Variable } & \multicolumn{2}{|c|}{ Unadjusted } & \multicolumn{3}{|c|}{ Adjusted } \\
\hline & OR & $p$ value & OR & $95 \% \mathrm{CI}$ & $p$ value \\
\hline Platelets & 0.986 & 0.085 & 0.985 & $0.968-1.003$ & 0.097 \\
\hline Direct bilirubin & 1.077 & 0.015 & 1.076 & $1.012-1.144$ & 0.019 \\
\hline Total bilirubin & 1.057 & 0.023 & 0.878 & $0.637-1.210$ & 0.427 \\
\hline Albumin & 0.473 & 0.163 & 0.509 & $0.153-1.697$ & 0.272 \\
\hline Child-Pugh score & 1.266 & 0.058 & 1.099 & $0.713-1.692$ & 0.669 \\
\hline
\end{tabular}

OR, odds ratio; CI, confidence interval.

Table 4. Factors associated with mortality in patients who did not undergo liver transplantation

\begin{tabular}{|c|c|c|c|c|c|c|}
\hline \multirow{2}{*}{ Variable } & \multicolumn{3}{|c|}{ Unadjusted } & \multicolumn{3}{|c|}{ Adjusted } \\
\hline & HR & $95 \%$ CI & $p$ value & HR & $95 \% \mathrm{CI}$ & $p$ value \\
\hline Significant IPS & 4.576 & $1.530-13.684$ & 0.007 & 3.432 & $1.096-10.748$ & 0.034 \\
\hline Child-Pugh score & 1.271 & $1.046-1.545$ & 0.016 & 0.852 & $0.596-1.218$ & 0.381 \\
\hline Direct bilirubin & 1.101 & $1.034-1.172$ & 0.003 & 1.074 & $1.003-1.150$ & 0.041 \\
\hline Hemoglobin & 0.741 & $0.589-0.932$ & 0.010 & 0.857 & $0.651-1.127$ & 0.270 \\
\hline
\end{tabular}

HR, hazard ratio; CI, confidence interval; IPS, intrapulmonary shunt. 
up to 12 months after LT and time-dependent reversal of IPS was characteristic. The follow-up time from LT to repeated cTTE was the only independent variable associated with post-LT IPS grade, suggesting that consideration of a time component is absolutely necessary in the interpretation of the results of repeated contrast echocardiography after LT.

HPS has been a topic of interest in most clinical investigations dealing with pathological alterations in the vasculature in patients with advanced LC, which is quite conceivable considering the prognostic impact of HPS and potential reversibility after LT [14-16]. Up to $40 \%$ to $60 \%$ of LC patients with normal levels of arterial blood gases have a positive contrast echocardiogram. This suggests that mild IPS, which is insufficient to alter gas exchange, is common $[5,17]$. Thus, confining HPS has the potential drawback of inability to evaluate the full clinical spectrum of pathological pulmonary vascular alterations in LC. For comprehensive assessment of the clinical impact of pathological alterations in the pulmonary vasculature, we evaluated IPS severity before and after LT and sought to investigate the chronology of IPS reversibility.

It is conceivable to expect lower $\mathrm{CaO}_{2}$ in patients with significant IPS, who definitely have a larger amount of

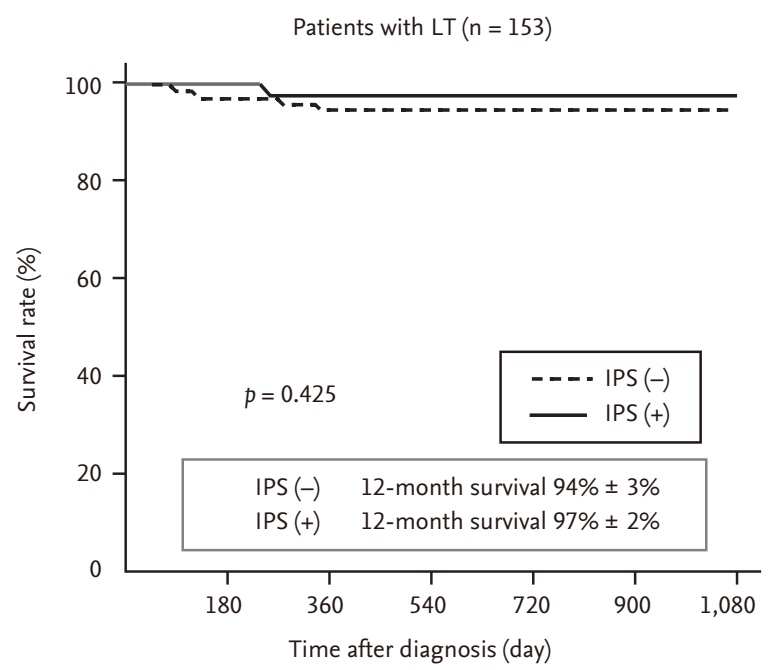

right-to-left shunt. Thus, decreased $\mathrm{CaO}_{2}$ is more like to be a result, rather than a cause of significant IPS. However, an interesting relationship between age and $\mathrm{CaO}_{2}$ has been observed in our study. Both young age and lower $\mathrm{CaO}_{2}$ were associated with significant IPS in unadjusted analysis (Table 2), but after adjustment, only $\mathrm{CaO}_{2}$ remained an independent variable. In patients with pulmonary arteriovenous malformation and normal liver function, $\mathrm{CaO}_{2}$ was reported to decrease with age in a serial observational study [18]. Our data are not sufficient to evaluate whether LC patients have different relationship between age and $\mathrm{CaO}_{2}$, but it can be an interesting research hypothesis. Decreased precapillary arteriolar tone resulting in vasodilation and angiogenesis are two potential mechanisms for IPS in LC patients. Numerous humoral factors including nitric oxide, endothelin, tumor necrosis factor, and vascular endothelial growth factor, were reported to be associated with pulmonary vasodilation and angiogenesis in an animal model of HPS [2]. Although the precise mechanisms are not known, the evidence supporting involvement of many vasoactive and growth factors suggest that IPS development is a metabolically active process. Further investigation is necessary to find the precise molecular mechanisms of IPS development and the impact of age

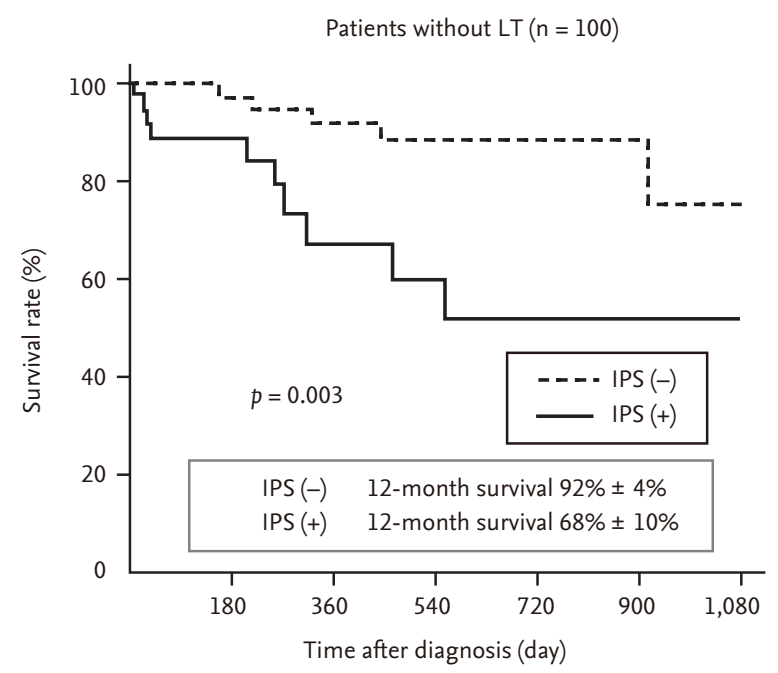

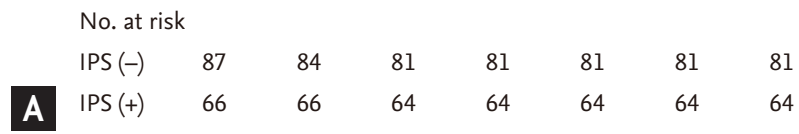

Figure 5. Impact of significant intrapulmonary shunt (IPS) on survival rate in patients (A) with and (B) without successful liver transplantation (LT). 
on this pathway.

In the literature, there were few clinical studies using repeated CTTE, from which limited information regarding the chronology of IPS or HPS reversibility after LT could be obtained [19,20]. The prevalence of IPS in pediatric patients who underwent LT was reported to be $16.8 \%$ (18/107) and IPS showed complete regression in all patients during follow-up in one study [19]. The mean duration of IPS was reported 5.8 months (range, 1 to 16 ). This study showed higher post-LT morbidity and mortality in patients with IPS than those without IPS. The authors struggled with the prognostic impact of early assessment of IPS status. However, several issues need to be considered before general application of these observations to other patient groups. First, the patient ranged in age from 9 months to 16 years, with a median age of 2 years. Compared to adults, the subjects were exceptionally young and the prevalence of IPS was relatively low. More importantly, two-thirds (12/18 patients) showed only mild grade 1 IPS and six patients had significant IPS (IPS grade 2, 3, and 4). This distribution is quite different from that observed in adult LC patients. Inclusion of small numbers of patients with significant IPS seems to be another inherent limitation of the study.

Repeated cTTTE and arterial blood gas analysis were done in 31 adult patients after successful LT and 100\% of the HPS cases reversed within the first year post-LT [19]. This is consistent with other studies showing a $100 \%$ reversal rate in patients with HPS 6 to 18 months following LT $[21,22]$. In this study, HPS reversed in $95.8 \%$ of the cases at 6 months, suggesting very rapid reversal of HPS after LT. However, the prevalence of negative cTTE, suggesting absence of IPS, was $69.2 \%$ at 6 months and $85.3 \%$ at 12 months. This represents a difference between HPS and IPS reversibility. Thus, HPS reversibility does not necessarily mean absence of IPS and our data showed that, although mild, persistent IPS was observed even 2 years after uneventful LT. These observations may support potential advantages of expansion of inclusion criteria from HPS to IPS for better appreciation of the pathological alterations of the pulmonary vasculature in LC patients. It is interesting to see that significant IPS could not be observed 12 months after LT in our study, showing similar chronology of HPS reversibility after LT [20]. After adjustment for pre-LT IPS grade, we con- firmed time-dependent reversal of IPS after LT. However, our study could not explain why there might be a certain cutoff time point. It is impossible to determine which of the potential mechanisms of IPS, pulmonary arteriolar vasodilation and angiogenesis, is more susceptible to or dependent on a time factor. Considering the relatively long time duration, up to 12 months after LT, for near complete reversal of HPS and significant IPS, it is likely that normalization of conventional parameters of underlying liver function is not an adequate condition for IPS reversibility. Further investigations are necessary to understand the molecular mechanism for time-dependent reversal of IPS.

The prognostic impact of significant IPS needs to be re-defined. In a prospective, multicenter US study, no difference in severity of liver disease in patients with or without HPS was observed [23]. Patients with and without HPS did not significantly differ in LT waiting list survival or post-LT survival [20]. In our study, direct bilirubin level was the only variable associated with development of HPS. Additionally, Child-Pugh score was independently associated with significant IPS, suggesting that severity of liver disease is the main factor determining IPS and HPS. This was well demonstrated by the finding that, if LT could not be performed, patients with significant IPS showed lower survival rate than those without (Table 4 and Fig. $5 \mathrm{~B}$ ). These observations may indicate that factors normally produced or metabolized in the liver could influence the lung microvasculature in susceptible individuals when hepatic function is altered [2]. The potential for preoperative testing for the severity of IPS to stratify patients for LT waiting list should be investigated.

As a retrospective analysis, there are several limitations in our study. Not all patients underwent cTTE after LT. Moreover, follow-up cTTE was not done at pre-specified intervals and the intervals between LT and follow-up cT'TE were too broad (6 to 952 days). Although pre-LT IPS grade or severity of individual patient was adjusted to assess the relationship between post-LT IPS grade and follow-up duration after LT, which may support that our main contention is acceptable, a prospective study with repeated follow-up cTTE after LT is warranted. Use of an arbitrary definition of significant IPS needs further explanation. As a truly quantitative classification of IPS is impossible, certain cutoff 
value of IPS grade has been used in clinical studies. We excluded mild (grade 1) to moderate IPS (grade 2) from the definition of significant IPS, resulting in more strict definition of significant IPS in our study compared with other studies. Less than 50\% of the patients (115/253) underwent arterial blood gas analysis and thus the real prevalence of HPS and its prognostic impact might not been adequately addressed.

In conclusion, significant IPS is a common complication of LC patients waiting for LT and is reversed after LT. Child-Pugh score representing underlying liver function, $\mathrm{CaO}_{2}$, and follow-up duration after LT were independently associated with pre- and post-LT IPS severity, respectively. These findings may support the idea that IPS development is an active metabolic pathway and these factors should be considered in the interpretation of IPS.

\section{KEY MESSAGE}

1. Before liver transplantation (LT), higher ChildPugh score and $\mathrm{CaO}_{2}$ are associated with significant intrapulmonary shunt (IPS) and successful LT resulted in decrease of frequency of significant IPS. Follow-up duration was the only variable associated with persistent IPS after LT.

2. Repeated contrast echocardiography was useful to evaluate factors associated with IPS. Consideration of follow-up duration after LT is critical in interpretation of contrast echocardiography test after LT.

\section{Conflict of interest}

No potential conflict of interest relevant to this article was reported.

\section{Acknowledgments}

The authors would like to thank professor Sung-Cheol Yun for statistical assistance.

\section{REFERENCES}

1. Rodriguez-Roisin R, Krowka MJ. Hepatopulmonary syndrome: a liver-induced lung vascular disorder. N Engl J
Med 2008;358:2378-2387.

2. Zhang J, Fallon MB. Hepatopulmonary syndrome: update on pathogenesis and clinical features. Nat Rev Gastroenterol Hepatol 2012;9:539-549.

3. Machicao VI, Balakrishnan M, Fallon MB. Pulmonary complications in chronic liver disease. Hepatology 2014;59:1627-1637.

4. Krowka MJ, Cortese DA. Hepatopulmonary syndrome. Current concepts in diagnostic and therapeutic considerations. Chest 1994;105:1528-1537.

5. Abrams GA, Jaffe CC, Hoffer PB, Binder HJ, Fallon MB. Diagnostic utility of contrast echocardiography and lung perfusion scan in patients with hepatopulmonary syndrome. Gastroenterology 1995;109:1283-1288.

6. Kim BJ, Lee SC, Park SW, et al. Characteristics and prevalence of intrapulmonary shunt detected by contrast echocardiography with harmonic imaging in liver transplant candidates. Am J Cardiol 2004;94:525-528.

7. Hopkins WE, Waggoner AD, Barzilai B. Frequency and significance of intrapulmonary right-to-left shunting in end-stage hepatic disease. Am J Cardiol 1992;70:516-519.

8. Lee JM, Choi MS, Lee SC, et al. Prevalence and risk factors of significant intrapulmonary shunt in cirrhotic patients awaiting liver transplantation. Taehan Kan Hakhoe Chi 2002;8:271-276.

9. Santa-Cruz RA, Pearson MD, Cohen MG, et al. Clinical predictors and characteristics of patients with chronic liver disease and intrapulmonary shunts. Clin Cardiol 2005;28:437-441.

10. Langiulli M, Aronow WS, Das M, et al. Prevalence and prognosis of intrapulmonary shunts in patients with hepatic cirrhosis. Cardiol Rev 2006;14:53-54.

11. Krowka MJ, Mandell MS, Ramsay MA, et al. Hepatopulmonary syndrome and portopulmonary hypertension: a report of the multicenter liver transplant database. Liver Transpl 2004;10:174-182.

12. Motomura T, Ikegami T, Mano Y, et al. Living donor liver transplantation for end-stage liver disease with severe hepatopulmonary syndrome: report of a case. Surg Today 2011;41:436-440.

13. Chen K, Li B. Reversal of severe hepatopulmonary syndrome in chronic hepatic cirrhosis by living donor liver transplantation: report of two cases. Surg Today 2011;41:441-443.

14. Stoller JK, Lange PA, Westveer MK, Carey WD, Vogt D, Henderson JM. Prevalence and reversibility of the hepa- 
topulmonary syndrome after liver transplantation. The Cleveland Clinic experience. West J Med 1995;163:133-138.

15. Krowka MJ, Porayko MK, Plevak DJ, et al. Hepatopulmonary syndrome with progressive hypoxemia as an indication for liver transplantation: case reports and literature review. Mayo Clin Proc 1997;72:44-53.

16. Gupta S, Castel H, Rao RV, et al. Improved survival after liver transplantation in patients with hepatopulmonary syndrome. Am J Transplant 2010;10:354-363.

17. Martinez GP, Barbera JA, Visa J, et al. Hepatopulmonary syndrome in candidates for liver transplantation. J Hepatol 2001;34:651-657.

18. Rizvi A, Macedo P, Babawale L, et al. Hemoglobin is a vital determinant of arterial oxygen content in hypoxemic patients with pulmonary arteriovenous malformations. Ann Am Thorac Soc 2017;14:903-911.

19. Kim JS, Kim KM, Ko JK, Lee YJ, Lee SG. Intrapulmonary shunt in the course of pediatric liver transplantation.
Transplant Proc 2008;40:2512-2514.

20. Pascasio JM, Grilo I, Lopez-Pardo FJ, et al. Prevalence and severity of hepatopulmonary syndrome and its influence on survival in cirrhotic patients evaluated for liver transplantation. Am J Transplant 2014;14:1391-1399.

21. Schiffer E, Majno P, Mentha G, et al. Hepatopulmonary syndrome increases the postoperative mortality rate following liver transplantation: a prospective study in 90 patients. Am J Transplant 2006;6:1430-1437.

22. Martinez-Palli G, Gomez FP, Barbera JA, et al. Sustained low diffusing capacity in hepatopulmonary syndrome after liver transplantation. World J Gastroenterol 2006;12:58785883.

23. Fallon MB, Krowka MJ, Brown RS, et al. Impact of hepatopulmonary syndrome on quality of life and survival in liver transplant candidates. Gastroenterology 2008;135:1168-1175. 